$10.2478 / \mathrm{gb}-2021-0004$

sciendo

\title{
Schreiben mit der Schere: Herta Müllers Collagenband Vater telefoniert mit den Fliegen
}

\section{Grazziella PREDOIU (Temeswar)}

Doz. Dr., West-Universität Temeswar;

E-Mail: grazziella_predoiu@yahoo.de

\begin{abstract}
Herta Müller has written several volumes of postcards, describing the work on the collages as a "relaxation exercise" from the laborious epic work. Her collage work is heterogeneous, works with the principle of chance, deals with clippings. The author tries o writing with scissors that can be positioned somewhere between literature and the fine arts. The volume Father Telephones with the Flies enables a political reading in which an I speaks about his traumas during the dictatorship, about interrogations, shadows and also includes the family sphere. Image and writing complement each other.
\end{abstract}

Keywords: Collages, hazard, political reading, dictatorship, interrogations, association between images and writing.

\section{Einleitung}

Herta Müller hat ein breit gefächertes Oeuvre verfasst, das im Zeichen der Autofiktionalität steht, welches den Bogen vom schwäbischen Frosch der Niederungen bis zum Frosch des Diktators spannt und auch den bundesdeutschen „Frosch der Freiheit" aus dem Westen miteinbezieht. Die Nobelpreisträgerin macht „die eigene Biografie zum Material eines fortgesetzten Nachdenkens über die alltäglichen Demütigungen, Bedrohungen und Zerstörungen des Individuums im Zeichen des 
menschenverachtenden Totalitären. "1 Ihr Werk umfasst Kurzprosa, die Romane der Ceaușescu-Trilogie, aber auch Collagen, die in avantgardistischer Manier mit bereits vorhandenem Sprachmaterial arbeiten. Darin setzt die rumäniendeutsche Autorin ein Schreiben mit der Schere ein, hantiert mit Ausgeschnittenem, mit dem Rissigen, das mit dem Visuellen assoziiert wird. Diesen Weg vom epischen Klein- und Großformatigen zur Text-Bild-Collage vollzieht die Nobelpreisträgerin nicht spontan, sondern in Sprüngen. Wenn ihr erster poetologischer Band Der Teufel sitzt im Spiegel. Wie Wahrnehmung sich erfindet Bilder enthielt, die einen Bezug zum Schreibprogramm der Autorin thematisierten und Collagen sich mit Essays abwechseln, so wird in der Erzählung Reisende auf einem Bein die Collage zur Möglichkeit, den Zustand der Fremde und Heimatlosigkeit zu überbrücken. Es folgen in zeitlichen Abständen mehrere Postkarten-Bände: Der Wächter nimmt seinen Kamm. Vom Weggehen und Ausscheren (1993), Im Haarknoten wohnt eine Dame (2000), Vater telefoniert mit den Fliegen (2013) und zuletzt Im Heimweh ist ein blauer Saal (2019). ${ }^{2}$ Ist Müllers Collagenwerk auf den ersten Blick von „Heterogenität ${ }^{\text {" }}$ ge-

${ }^{1}$ Eke, Otto Norbert: Schönheit der Verwund(er)ung. Herta Müllers Weg zum Gedicht. In: Text + Kritik. Herta Müller. hrsg. Heinz Ludwig Arnold Nr 155/2002, München 2002, S. 64-80, hier 65.

2 Müller hat ihre Collagen nicht nur in den vier erwähnten Bänden publiziert, sondern auch in wissenschaftlichen Publikationen, sie hat auch einen Collagenband in rumänischer Sprache veröffentlicht, Este sau nu este Ion. Iași 2005. Im 2002 verlegten Band Text und Kritik, der Herta Müller gewidmet ist, gibt es außerdem Fünf Collagen, die Herta Müller zusammengestellt hat. Sie fügte 2013 der Publikation von Bettina Brandt/Valentina Glajar (Hrsg.): Herta Müller. Politics and aesthetics Lincoln 2003 neue Collagen unter dem Titel Collage poems ein.

${ }^{3}$ Rossi, Christina: Vom Trauma zum Tabu. Schweigen und Subversion. In: Deeg, Jeens Christian/ Wernli, Martina (Hrsg.): Herta Müller und das Glitzern im Satz. Eine Annäherung an Gegenwartsliteratur. Würzburg: Königshausen u. Neumann 2016, S. 237-261, hier 244. 
prägt, weist es trotz des umfangreichen Arbeitszeitraums und des inhaltlichen Rahmens trotzdem strukturelle Ähnlichkeiten auf. Zeichnen die Collagen der ersten beiden Bände das Bild einer bruchstückhaften und lückenhaften Welt, noch befremdliche Sprachbilder, kann in den neueren Collagen-Bänden ein Wandel festgestellt werden - weg von der starken Textdominanz und der harmonischen Farbigkeit hin zu einer Betonung der einzelnen Schriftelemente in Größe, Farbigkeit und Typ und deren Wechselspiel mit den Bildern.

Die Autorin hat das Erstellen der Collagen als eine Art handwerkliche „Entspannungsübung“ zwischen der Arbeit an den breit gefächerten Romanen dargestellt. Im Essayband Der König verneigt sich und tötet unterstreicht sie, dass sie Collagen angefertigt habe, um ihren Freunden von Reisen Persönliches mitzubringen, wobei die ersten Collagen im Zug erstellt wurden:

Beim Zeitungslesen im Zug klebte ich ein Bildfragment und Wörter zusammen auf einer weißen Karte oder, ein zwei Sätze [...] Erst die Verblüffung, was lose Zeitungswesen alles hergeben, brachte das Reimen mit sich. Ich schnitt schon lange auch zu Hause Wörter aus. Wahllos, wie mir schien, lagen sie auf dem Tisch. Ich sah sie mir an, und erstaunlich viele reimten sich. [...] nahm ich die Reime an, für die ich nichts getan habe, die sich zufällig auf der Tischplatte getroffen hatten. Es waren Worte, die einander kennenlernten, weil sie sich den Ort, wo sie lagen, teilen mussten. Ich konnte sie nicht wegjagen und kam auf den Geschmack des Reimens. ${ }^{4}$

Sie führt die Arbeit an Collagen mit ihrer Erfahrung in einer Diktatur in Verbindung, mit dem Papier-Sparen in Rumänien, mit dem Mangel an bunten Zeitschriften,

Die ganze Kleberei hat [...] mit meiner früheren Zeit in Rumänien zu tun. Dass es unzählige bunte Zeitschriften gibt, so gutes Papier, so viele Texte, die nur flüchtig gelesen und schon weggeschmissen

${ }^{4}$ Müller, Herta: Der König verneigt sich und tötet. Frankfurt 2008, S. 56. 
werden - das alles kannte ich in Rumänien nicht. Es gab nur graue, nach Schmieröl stinkende Staatszeitungen, sonst nichts. ${ }^{5}$

und setzt sie einem Freiheitsrausch gleich, hatte sie doch die Erfahrung gemacht, dass man in einer Diktatur Geschriebenes vor dem wachsamen Auge der Macht verbergen musste: „Dass sie [die Wörter] offen herumliegen dürfen, ist für mich ein Ausdruck von Privatheit, von Ungezwungenheit, sogar von persönlicher Freiheit. Denn Wortbesitz im Überfluss ist das Gegenteil von früher, von Zensur."

Müller hat die Arbeit an den Collagen als ,Spielerei $[\ldots]^{67}$ aber auch als Befreiung von Schreib-Zwängen verstanden wissen wollen, als Experimentierfeld zur Überwindung totalitärer Beschädigungen, doch auch das Experiment der Collagen kann nicht von den Schrecken der Geschichte losgelöst werden, die Grunderfahrung der Angst bleibt erhalten. Ihre Collagen sind thematisch eng an die Romane angelehnt, es lassen sich dieselben Themen herausarbeiten: die nationalsozialistische Vergangenheit der Rumäniendeutschen, die rumänische Diktatur, die Unterdrückung, sexueller Missbrauch, Selbstmord, Flucht und Auswanderung.

\section{Gattungszuordnung und Wesensmerkmale:}

Die Postkarte ist in der Literatur ein junges Medium. Wesentlich jünger als der Brief, - sie wurde 1869 erfunden -, dient sie dazu, kurze, nicht-geheime Botschaften zu senden. Damit steht sie im Zeichen der Modernität nicht nur aufgrund ihres jüngeren Erscheinungsdatums, sondern auch wegen der spezifischen Eigenschaften im Kommunikationsprozess. Sie leistet „ihren

5 Müller, Herta: „In vielen Wörtern sitzt was Rumänisches“ In: Welt. Kultur vom 13.03.2019, unter: https://www.welt.de/kultur/literarischewelt/article190250951/Wortbesitz-gegen-die-Zensur-HertaMueller-ueber-ihre-Collagentechnik.html [Zugriff am 1.11.2020].

6 Ebenda.

${ }^{7}$ Müller, Herta: Im Heimweh ist ein blauer Saal. München 2019, [o. S.]. 
Dienst nur in Gesellschaften mit hoher Mobilität und ausgeprägten Infrastrukturen zur Kommunikation. ${ }^{\text {“8 }}$ Dabei schlägt die Postkarte die Brücke zur bildenden Kunst und ist in drei Varianten aufzufinden: die Kunstpostkarte, die Bildpostkarte, der selbstständige künstlerische Darstellungsformen zugrunde liegen; und die Postkarte als eigenständiges Kunstwerk, welche als Übermittlerin von Bildmitteilungen genutzt wird. ${ }^{9}$ Im Spannungsfeld zwischen Literatur und Visuellem sind auch die Collagen Müllers zu verorten.

Collagen gehen vom gegebenen Wort-Material aus, das zweckdienlich entfremdet und anderswo eingebaut und montiert wird, wobei wie im Falle der Anagramme der Text im Ausgangsmaterial selbst angelegt ist. Das disparate Material, das meistens aus den Medien stammt, wird fragmentarisiert und zu einer neuen Einheit zusammengefasst. Die Montage ist dabei zweifach codiert: ,die in einen aufnehmenden Zusammenhang eingefügten [...] Montage-Fragmente verweisen auf ihre Herkunft und sind zugleich Elemente des neuen Zusammenhangs." ${ }^{\prime 10}$ Herta Müller übernimmt bereits veröffentlichte Wörter, sie schneidet sie aus Zeitschriften aus und setzt sie neu zusammen, reduziert sie manchmal auf die kleinste semantische Einheit:

Die Worte der Collage müssen einem gar nicht einfallen, sie liegen alle gleichzeitig auf dem Tisch. Es ist eine andere Entscheidung, aus dem Vorhandenen zu nehmen, als wenn man Wörter aus dem Kopf schreibt. Es ist alles gleichzeitig da, man schiebt sie mit der Hand hin und her. ${ }^{11}$

8 Müller, Julia: Sprachtakt. Herta Müllers literarischer Darstellungsstil. Köln/Weimar 2014, S. 209.

9 Vgl. Ebenda, S. 210.

${ }^{10}$ Möbius, Hanno: Collage oder Montage. In: Hubert van den Berg/ Walter Fähnders (Hgg.): Metzler Lexikon Avantgarde. Stuttgart/ Weimar 2009, S. 65-67, zit nach Eke, Norbert Otto (Hg.): Herta Müller. Stuttgart 2009. S. 72.

${ }^{11}$ Eddy, Beverley Driver: „Die Schule der Angst“. Gespräch mit Herta Müller, 14. April 1998. In: The German Quarterly 1999, H. 4, S. 329339, hier 338. 
Dem Zufall kommt dabei eine entscheidende Rolle zu, die Collagen entstehen vor den Augen der Schriftstellerin:

Ich könnte nie ein Gedicht schreiben, mich hinsetzen, mit der Hand und mit dem Stift. Aber [...] wenn ich diese gedruckten Wörter ausgeschnitten auf dem Tisch liegen habe, fliegen sie zusammen und werden eine Geschichte. Sie lassen mich draußen und ich bin noch drin [...]. Da sieht man noch mehr, als wenn man aufs Papier schreibt, wie sehr alles dem Zufall gehorcht. Sicher weiß man, was man will, aber im Augenblick, wenn man's tut, wird es Zufall. ${ }^{12}$

Diese akribische Arbeit an und mit der Sprache setzt einen leidenschaftlichen Umgang mit sprachlichen Elementen voraus, „weil man jedes Wort einzeln anfassen muss. Überhaupt ist diese Arbeit sinnlich. Und sie ähnelt in vielem dem wirklichen Leben: Der Zufall, durch den sich die Wörter treffen." ${ }^{13}$ Das Verschieben und Neuordnen von ausgeschnittenen Bruchstücken enthält ein gewisses Ausmaß an Zufälligkeit, es wird zum „,iterarischen Handwerk ${ }^{\text {"14 }}$, das ,den Produktionsprozess, die Bildmontage und die Wort-Fügung, visuell ausstellt. " ${ }^{15}$

Die willkürliche Kombination von vorgefundenem Material, der Zufall als Selektionsprinzip verbindet diese Texte mit der surrealistischen und dadaistischen Tradition, die in der Absicht, in der zufälligen sprachlichen Fügung eine neue Wahrheit aufblitzen zu lassen, Wörter und Bilder aus ihrem ursprünglichen Zusammenhang lösten und sie möglichst zufällig neu zusammensetzten, wobei sie aleatorische Prinzipien des nicht gesteuerten Schreibens erprobten. Verbindungslinien können bis zur Anagrammproduktion des befreundeten Poeten Oskar Pastior hergestellt werden. Die Autorin hortet Wörter auf ihrem

\footnotetext{
${ }^{12}$ Ebenda, S. 338.

${ }^{13}$ Müller, In vielen Wörtern..., unter: https://www.welt.de/kultur/literarischewelt/article190250951/Wortbesitz-gegen-die-Zensur-HertaMueller-ueber-ihre-Collagentechnik.html [Zugriff am 1.11.2020]

${ }^{14}$ Ebenda, S.338.

${ }^{15}$ Eke 2002, S. 71.
} 
Arbeitstisch und gewährt diesen eine gewisse Eigendynamik, was an das Verfahren der écriture automatique der historischen Avantgarde ${ }^{16}$ erinnert: „Weil die Wörter schon vorhanden sind, denke ich manchmal, dass ich es gar nicht bin, die schreibt. " ${ }^{17}$ Trotz der avantgardistischen Verdichtungs- und Verfremdungstechniken ist es die kontrollierende Instanz der Autorin, die den „Prozess ansetzt, steuert und selbst bestimmt, in welchem Zustand das Resultat mit Klebstoff und aus der Hand gehen wird. " ${ }^{18}$ Ihre Collagen sind also dennoch nicht dem surrealistischen ecriture automatique verhaftet, die Kontrolle durch die Ratio lässt sich nicht vollkommen ausschalten.

\section{Vater telefoniert mit den Fliegen (2012)}

Der 2012 im Hanser Verlag publizierte Band Vater telefoniert mit den Fliegen ${ }^{19}$ besteht aus 5 Teilen und umfasst 187 Collagen, wobei im Vergleich zu früheren Bänden weniger die Farbe und Formgebung prägnant sind, sondern es werden hier eher einzelne Schriftelemente betont und wie diese vom Typ, der Farbe und der Größe her mit dem Sprachlichen in ein Wechselspiel treten. Das Format des Buches verstärkt die Rezeption der Collagen im Sinne künstlerisch-literarischer Werke, wobei die

${ }^{16}$ Vgl. Bürger, Peter: Theorie der Avantgarde. Frankfurt/Main: Suhrkamp 1974.

${ }^{17}$ Müller, Herta: „Ich habe die Sprache gegessen. “ Die Literatur-Nobelpreisträgerin Herta Müller über ihre zusammengeklebten Gedichte und über die Macht und das Versagen der Wörter. In: Der Spiegel Nr. 35, 2012, S. 128-132, hier 140.

${ }^{18}$ Mitterbauer, Helga: Ästhetische Hybridisierung: Ästhetische Verfremdungstechniken in Herta Müllers Die blassen Herren mit den Mokkatassen. In: Paul Michael Lützeler/ Erin McGlothin (Hgg.): Gegenwartsliteratur. Ein germanistisches Jahrbuch. Herta Müller, Bd. 10, Tübingen 2011, S. 75-93, hier 84.

${ }^{19}$ Müller, Herta: Vater telefoniert mit den Fliegen. Frankfurt: Fischer 2014. Des Weiteren wird im Verlaufe des Beitrags die Sigle VF für den Collagenband eingesetzt. 
Verschiebung vom Künstlerischen zum Literarischen ${ }^{20}$ bemerkbar ist: Es betont deren lyrischen Charakter und fungiert als vereinendes Medium, das sowohl künstlerische als auch literarische Werke präsentiert. Der Band besitzt Seitenzahlen, ist in fünf ungefähr gleich lange Abschnitte unterteilt, deren Benennung durch ein Zitat aus der Collage am jeweiligen Beginn des Abschnitts erfolgt. Genau wie in den vorherigen Bänden besitzt jede Karte eine eigene Titelseite, sodass es dem Leser frei steht, die Sammlung als geschlossenes Werk zu deuten, mit einem fortlaufenden, in Teilen voneinander abhängigen Text oder jede Karte als geschlossenes Artefakt zu deuten. Die Collagen sind gemäß den Gepflogenheiten der Gattung Lyrik in Versform verfasst, obwohl viele von ihnen keinen Reim und Rhythmus aufweisen.

Die Themen kreisen um dörfliche und städtische Welt, um Auswanderung, Grenze und Flucht, Verrat bis zu Angst, Verhören, Suizid, Tod der engsten Freunde, ${ }^{21}$ zum Motiv der Freundschaft, um Figuren wie Vater und Mutter, der Nachbar, der Wachmann, der Polizist. Die Autorin ,macht die eigene Biografie immer wieder zum Material eines fortgesetzten Nachdenkens über die alltäglichen Demütigungen, Bedrohungen und Zerstörungen des Individuums im Zeichen des menschenverachtenden Totalitären.“22

Ohne Rückbezug auf frühere Texte und Szenerien bleiben die Collagen schwer verständlich. Es sind überwiegend bedrohliche Situationen und düstere Themen, wie es einige Beispiele

${ }^{20}$ Vgl. Müller Julia 2014, S. 220.

${ }^{21}$ Auch auf diesen Band trifft die Überlegung Otto Norbert Ekes zu, dass diese düsteren Themen ihren ,geheimen Fluchtpunkt“ in den verschiedenen Ausprägungen des Todes finden: als physischer Tod (auf der Flucht oder als Selbstmord wegen den Verhören), als moralischer Tod (durch den Verrat, den Opportunismus und die Feigheit im Umgang mit der Macht) und als gesellschaftlicher Tod (im Stillstand unter der Erstarrung). In: Eke 2002, S. 71.

${ }^{22}$ Ebenda, Eke 2002, S. 65. 
bezeugen können: „AN DER Grenze hat mich der/Wachmann gefragt“ (VF 12), „Mutter sagt am Telefon der Holunder/ blüht sich müd zwei Sommer“ (VF 21), „Der Mann vom Geheimamt folgt" (VF, 22), „Ach, ein so leichtes/ Fleisch hat der Verrat" (VF 13), ,der Tod ist/ eine schmale/ kahle Meterware“(VF 31), „dass mich/ die kleine/ Heimat die/ es je gab/ familiär leergefressen hatte“ (VF 38), „Der umgebrachte Freund darf/ gratis im Nest der Gräser übernachten“ (VF 39), „,nichts als ein Hase/ im Hals war die Angst“ (VF 57), ,als ich vom Verhör kam/ war ich niemandes Kind“ (VF 69), „Mutter schiebt ein Bonbon/ im Mund hin und her/ Vater telefoniert mit den Fliegen" (VF 77). Das Ich befindet sich in beklemmenden Situationen, an der Grenze, sehr oft beim Verhör, als reflektierende Instanz im Dorf mit Mutter und Vater, wobei Machtdiskurse innerhalb der Familie, der Geschlechter und innerhalb des die Gesellschaft durchdringenden Systems anzutreffen sind. Durch öfters eingesetzte Begriffe wie „Kohle“, „Schnee“, „Pappeln“ und „Mais“ werden Verbindungslinien zu Müllers schriftstellerischem Werk, hauptsächlich zur Atemschaukel, kenntlich. „Das sprechende Subjekt beschränkt sich stets auf die erste Person Singular" ${ }^{\text {“23, }}$ „ich sage“, tritt aber auch in Dialog mit „er sagt", wobei in vielen Collagen eine Form des Dialogs anzutreffen ist. In dieser Welt herrscht keine Freundschaft, kein Gemeinschaftsgefühl, das durch ein „Wir“ reflektiert werden könnte. Sehr viele Collagen basieren auf einer Monolog- oder Dialogform, wobei die Kommunikation mitreflektiert wird:

Die Wiedergabe menschlicher Kommunikation wird zum zentralen Thema der Collagen und erhält auch formal die Bedeutung szenischen Geschehens, wenn es in einzelnen Collagen lediglich ein Satz oder ein kurzer Dialog ist, der den ganzen Inhalt der Collage bildet. Das gesprochene Wort ersetzt dann das Handeln. ${ }^{24}$

${ }^{23}$ Müller, Julia 2014, S. 214.

${ }^{24}$ Rossi 2016, S. 246. 
Neben semantisch offenen Texten gibt es auch hermetisch-verschlüsselte, sodass sich Deutungsversuche dem Vorwurf der Spekulation aussetzen.

Bildteile stehen gleichberechtigt neben den Textelementen. „Auf jeder Karte steigt der Text mit dem Bild auf eine Bühne, jede Karte inszeniert ihr kleines Theater ${ }^{625}$, schreibt Herta Müller in ihrem letzten Collagenband. Dabei treten die Texte mit den Bildelementen in Beziehung, sodass der Bildteil einen im Text erwähnten Gegenstand darstellt, jedoch sind die ausgeschnittenen Bildeinzelteile nicht genau herausgetrennt. Sehr oft präsentieren sie Körperteile oder menschliche Gesichter, wobei viele Ausschnitte aus Fotografien stammen. Vom Optischen her dominieren Körperteile, Fragmente von Menschen, Tieren und Gegenständen. Die Menschen sind meist sehr klein, Schatten, die über den Bildern schweben, manchmal hyperbolisch überdimensioniert. Menschliche Gesichter sind nicht kenntlich, fratzenhaft überdimensioniert oder verkleinert. Sie wirken meistens zerschnitten wie in den Collagen Picassos. Es gibt auch Beispiele, in denen die herausgeschnittene Form nichts mit der Ursprungsabbildung gemeinsam hat, da die einzelnen Ausschnitte durch ihre Farbgebung und durch die unterschiedliche Größe einen eigenständigen Bildteil ergeben. ${ }^{26}$ Schrift und Bild bleiben getrennte Bereiche und sind nicht miteinander vernetzt wie in der surrealistischen oder dadaistischen Collage, die Bildcollagen korrespondieren mit den Texten auf Abstand.

Die Beschädigung und die unbestimmten Ängste eines gespaltenen, meist weiblichen Ich, das mit alltäglicher und staatlicher Gewalt sowie mit dem Tod konfrontiert ist, spiegeln sich auch in diesem Band in Natur und Umgebung wider, was eine

${ }^{25}$ Müller Herta, In vielen Wörtern..., unter: https://www.welt.de/kultur/ literarischewelt/article190250951/Wortbesitz-gegen-die-ZensurHerta-Mueller-ueber-ihre-Collagentechnik.html [Zugriff am 1.11.2020].

${ }^{26}$ Vgl. Müller Julia 2014, S. 219. 
politische Lesart der Collagen zulässt. Die Collage auf Seite 12 bietet eine politische Lesart an und ist im Sinne der Biografie des fiktionalen Ichs zu deuten. Sie ist äußerlich in Verse eingeteilt, ist aber reimlos.

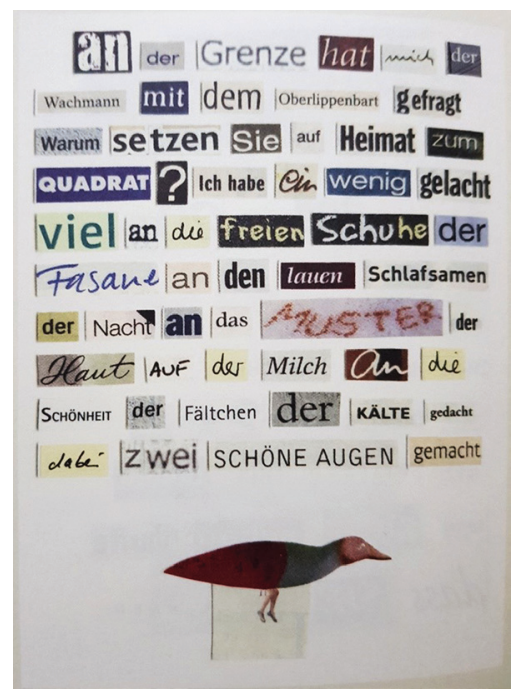

Auf den ersten Blick fällt das Fehlen der Interpunktion auf, das zu einem Kennzeichen des Bandes wird. Die ausgeschnittenen Wortteile und Wörter setzen sich zu einem Textblock ohne syntaktische und oft auch ohne semantische Regeln zusammen. So wird der „fragmentarische Charakter der Collagen konsequent gehalten und die herkömmliche Herangehensweise an einen lyrischen Text gestört. "'27 Größere Worte alternieren mit kleineren, es dominieren gedämpfte Farben, gelb, blau, rot, aber auch dunkle Farben. Die Collage präsentiert das lyrische Ich an der Grenze, die zusammen mit den Begriffen „Polizist“, „Wachmann“, „Koffer“"zu einem Topos der rumäniendeutschen Autorin

${ }^{27}$ Hedayati-Aliabadi, Minu: „Der fremde Blick““ - „ein fremdes Auge“. Transmediale Inszenierungen von Schrift und Bild in Herta Müllers Collagen. In: Textpraxis 5, 2.2012, unter: https://www.textpraxis.net/ minu-hedayati-aliabadi-transmediale-inszenierung-von-schrift-undbild-in-herta-muellers-collagen [Zugriff am 14.10.2020]. 
geworden ist. Opponiert wird dem Wachmann eine Ausreisende. Das lyrische Ich ist im Begriff, legal das Land zu verlassen und wird nach dem Ausreisewunsch gefragt. Ironisch wird dabei der Begriff „Heimat zum Quadrat“ (VF 12) eingesetzt, der auf die Unmöglichkeit einer doppelten Heimat verweist. Als Ausweichmanöver, um Zeit zu gewinnen oder aber als Strategie, um der Angst zu entkommen, ersinnt das lyrische Ich in einer metaphernreichen Sprache vier Freiräume. Es denkt an die „freien Schuhe der Fasane“ - erinnert werden soll dabei an den Erzählband Der Mensch ist ein großer Fasan auf der Welt, wobei die Hilflosigkeit des Fasans vor dem Jäger auf den Menschen übertragen wird; Die Wendung, ,den lauen Schlafsamen der Nacht" (VF 12) lässt sich im Sinne einer Wortschöpfung deuten, die auf die therapeutische Funktion der Nacht hinweisen könnte. „An das Muster der Haut auf der Milch“ (VF 12) eröffnet einen Deutungshintergrund in Richtung Kindheit, auch wenn diese für das fiktionale und nicht-fiktionale Ich mit Traumata verbunden ist. Schließlich überwiegt die Selbstsicherheit und die Wegfahrende denkt an die „Schönheit der Fältchen der Kälte" (VF 12) und lächelt, somit mündet die Collage nicht ins Grauen, sondern in eine vorgespielte Überlegenheit des Ich. Dass die Heimat für das Ich zum Schauerszenario wird, wird einige Seiten später betont, denn es wird unterstrichen, ,dass mich die kleine Heimat familiär leergefressen hatte." (VF 38). Schaut man sich das Bild an, so wird mittig der im Text erwähnte Fasan montiert, wobei dieser bewegungslos scheint. Wie in den vorherigen Collagenbänden bleiben Schrift und Bild getrennte Bereiche und verbinden sich nicht zu einer ineinander verwobenen Gesamtstruktur.

Verhör und Beschattungen ergeben einen weiteren Themenkreis: Die Collage auf Seite 69 bezieht sich auf die Situation nach einem Verhör, wobei ausschließlich die Perspektive des weiblichen Ich-Opfers eingenommen wird. Diesmal befindet sich der Text in der unteren Hälfte, während das Bild den 
oberen Teil einnimmt. Diese Collage wendet sich gegen die auf die psychische Verfassung des Subjekts Einfluss nehmende Machtstruktur und bedient sich dabei der Mechanismen subversiv arbeitender Diskurse.

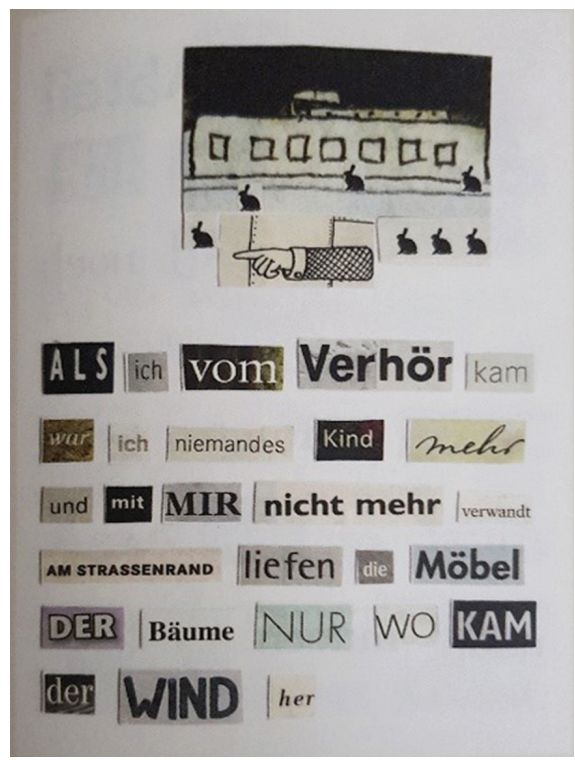

Die innere Leere nach dem Kontakt mit einem Machtelement, das den Raum hinter der Vernehmung besetzt, wird durch die Redewendung ,niemandes Kind mehr sein“ (VF 69) verdeutlicht, wiewohl auch auf die innere Verwahrlosung durch die Zeile „mit mir nicht mehr verwandt" hingewiesen wird. Die Begriffe erinnern an den Roman Heute wär ich mir lieber nicht begegnet, der auf der Grundlage einer Straßenbahnfahrt in Richtung Verhörstelle organisiert ist. Von der Schriftgröße und der gewählten dunklen Farbe her hebt sich das Substantiv „Verhör“ optisch von den anderen Wörtern ab, wodurch auf die für die Menschen zerstörende Funktion der Kreuzverhöre in einem totalitären Staat hingewiesen wird. Der inneren Verwüstung entspricht eine Entgleisung des Äußeren, das ins Surreale umkippt, „liefen die Möbel der Bäume“ (VF 69). Die Collage endet mit 
einer Frage, die als solche nicht markiert ist, ,wo kam der Wind her" (VF 69). Schaut man sich das montierte Bild an, so entspricht es etwa der dargestellten Situation, es bildet ein Gefängnis oder einen Untersuchungsraum ab, der statt von Menschen von Hasen umringt ist. Zwar fehlt der panoptische Blick, der alles einfängt, seinen Platz hat der mahnende Zeigefinger eingenommen, der vor dieser Form der psychischen Tortur warnt.

Die Wortschnipsel sind nicht mehr in der bestimmten Größe gehalten, sondern es tritt auch das „In-Szene-Setzen der Schrift“28 auf. Dank der inszenierten Schriftbausteine werden Inhalte vermittelt, die in der ursprünglichen Wortbedeutung nicht angelegt sind, was auf die Nähe der Collagen zum Anagramm verweist.

Wenn viele Collagen um das Thema Verhör, Angst und Verrat, Tod eines Freundes, Suizid kreisen, so gibt es in Vater telefoniert mit den Fliegen eine Erweiterung des Themenspektrums um den dörflichen und familiären Raum, denn Müllers fiktive Erzählerin greift zurück in ihre nicht vom Geheimdienst reglementierte Kindheit. Im Unterschied zu dem Band Niederungen, der den Bruch mit der familiären Welt potenziert, kann von einer versöhnenden Geste gesprochen werden, auch wenn Aggressivität diesem Raum innewohnt.$^{29}$ Es tauchen Figuren wie Mutter, Vater, der Uhrmacher, der Polizist, der Nachbar auf.

Die Ich-Figur spricht im kindlichen Ton, in einer dürftigen Sprache über die engsten Familienmitglieder, über patriarchalische Rollenzuschreibungen im Dorf(VF 136). Wenn die Mutter nur durch ihren Beruf als Mutter kenntlich gemacht wird, so wird der Vater über das Lkw-Fahren definiert, wobei wie in den Erzähltexten die Possessivpronomen „mein“, „meine“, die von der Nähe zu den Angehörigen zeugen, fehlen. Der kurze Satz „war er" markiert den Tod des Vaters, dessen Alkoholsucht den

${ }^{28}$ Ebenda.

${ }^{29}$ Vgl. dazu die Collage auf S. 111, in welcher die Mutter als ordnende Instanz erscheint und einen Hund mit einem Stein erschlägt, darauf stolz bekundet: ,jetzt ist er erledigt.“ 
Tod bewirkt hat. Der Blick gleitet auf das Haus, dessen Position durch die doppelte Neubildung, ,pelztief“ im Wechsel der Jahreszeiten definiert ist. Die Collage endet mit der Frage ,wer wechselt das", wobei das Satzzeichen fehlt.

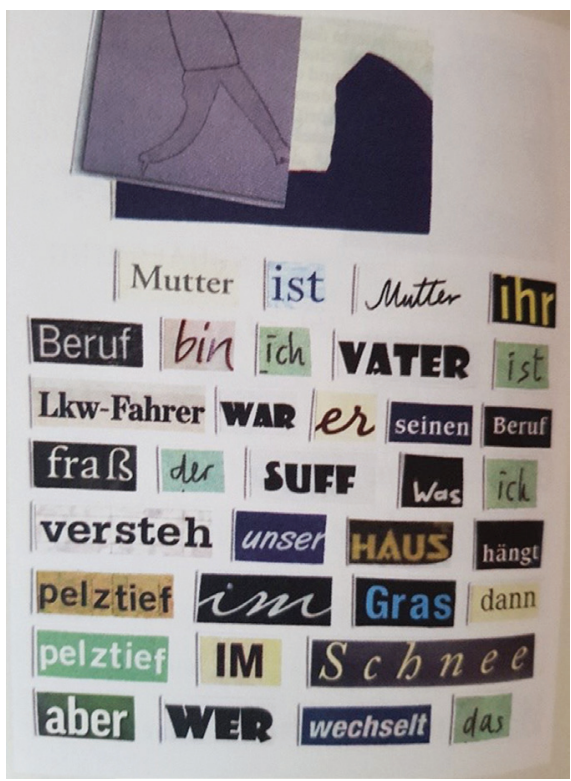

\section{Fazit:}

Das Postkartenbuch Vater telefoniert mit den Fliegen weist auf die gleiche Thematik hin, zu der auch in den Erzählungen und Romanen Herta Müllers Stellung genommen wird, die Auseinandersetzung mit dem totalitären Rumänien, wobei eine politische Lesart zur Entschlüsselung der Botschaft beiträgt. Die Collagen gehorchen dem Zufallsprinzip, sie setzen ein aleatorisches Schreibprinzip voraus und stellen eine Fortsetzung der Sprachexperimente unter Rekurs auf freie Schreibtechniken dar. Arbeitete Müller in ihren kurzen Prosatexten an einer Reduktion und Verschlankung des Stils, so bildet die Festlegung auf Collagen eine Form der reduzierten Beschränkung, der Verdichtung 
und Verknappung der Botschaft. Das Schreiben mit der Schere, die Arbeit mit dem Ausgeschnittenen, dem Bruchstückhaftem und dem Riss situiert Müller in die Traditionslinie der Avantgarde.

\section{Literaturverzeichnis}

\section{Primärliteratur:}

Müller, Herta: Der Wächter nimmt seinen Kamm. Vom Weggehen und Ausscheren. Reinbek bei Hamburg: Rowohlt 1993. Müller, Herta: Im Haarknoten wohnt eine Dame. Reinbek bei Hamburg: Rowohlt 2000.

Müller, Herta: Der König verneigt sich und tötet. Frankfurt: Fischer 2008.

Müller, Herta: Vater telefoniert mit den Fliegen. Frankfurt: Fischer 2014.

Müller, Herta: Im Heimweh ist ein blauer Saal. München: Hanser 2019.

Müller, Herta: „Ich habe die Sprache gegessen.“ Die LiteraturNobelpreisträgerin Herta Müller über ihre zusammengeklebten Gedichte und über die Macht und das Versagen der Wörter. In: Der Spiegel 2012, 35, S. 128-132.

\section{Sekundärliteratur:}

Brandt, Bettina/ Valentina Glajar (Hgg.): Herta Müller. Politics and aesthetics. Lincoln: University of Nebraska Press 2003.

Bürger, Peter: Theorie der Avantgarde. Frankfurt/Main: Suhrkamp 1974.

Eddy, Beverley Driver: „Die Schule der Angst“ - Gespräch mit Herta Müller, 14. April 1998. In: The German Quarterly 1999, H. 4, S. 329-339.

Eke, Otto Norbert: Schönheit der Verwund(er)ung. Herta Müllers Weg zum Gedicht. In Text + Kritik. Herta Müller. hrsg. Heinz Ludwig Arnold Nr 155/2002, München 2002, S. 64-80. 
Eke, Norbert Otto (Hrsg.): Herta Müller. Handbuch. Stuttgart: Metzler 2017.

Rossi, Christina: Vom Trauma zum Tabu. Schweigen und Subversion. In: Deeg, Jeens Christian/Wernli, Martina (Hgg.): Herta Müller und das Glitzern im Satz. Eine Annäherung an Gegenwartsliteratur. Würzburg: Königshausen u. Neumann 2016, S. 237-261.

Mitterbauer, Helga: Ästhetische Hybridisierung: Ästhetische Verfremdungstechniken in Herta Müllers Die blassen Herren mit den Mokkatassen. In: Paul Michael Lützeler/ Erin McGlothin (Hgg.): Gegenwartsliteratur. Ein germanistisches Jahrbuch. Herta Müller, Bd. 10, Tübingen 2011, S. 75-93.

Möbius, Hanno: Collage oder Montage. In: Hubert van den Berg/Walter Fähnders (Hgg.): Metzler Lexikon Avantgarde. Stuttgart/Weimar 2009, S. 65-67.

Müller, Julia: Sprachtakt. Herta Müllers literarischer Darstellungsstil. Köln/Weimar: Böhlau 2014.

\section{Internetquellen:}

Müller, Herta: „In vielen Wörtern sitzt was Rumänisches“ In: Welt. Kultur vom 13.03.2019, unter: https://www.welt.de/ kultur/literarischewelt/article190250951/Wortbesitzgegen-die-Zensur-Herta-Mueller-ueber-ihreCollagentechnik.html [Zugriff am 1.11.2020].

Hedayati-Aliabadi, Minu: „Der fremde Blick“ - „ein fremdes Auge“. Transmediale Inszenierungen von Schrift und Bild in Herta Müllers Collagen. In: Textpraxis 5, 2.2012, unter: https://www.textpraxis.net/minu-hedayati-aliabadi-transmediale-inszenierung-von-schrift-und-bild-in-herta-muellers-collagen [Zugriff am 14.10.2020]. 